\title{
Simulations of diamond nucleation in carbon fullerene cores
}

\author{
R. Astala, ${ }^{*}$ M. Kaukonen, and R. M. Nieminen \\ Laboratory of Physics, Helsinki University of Technology, P.O. Box 1100, FIN-02015, Finland \\ G. Jungnickel and Th. Frauenheim \\ Universität/Gesamthochschule Paderborn, 33095 Paderborn, Germany
}

(Received 1 November 2000; published 1 February 2001)

\begin{abstract}
Recent experiments have shown that heavy ion or electron irradiation induces the nucleation of diamond crystallites inside concentric nested carbon fullerenes, i.e., bucky onions. This suggests that the fullerene acts as a nanoscopic pressure shell. In this paper we study the formation of tetrahedrally bonded carbon inside a prototype icosahedral two-shell fullerene by means of atomic-scale computer simulations. After the simulated irradiation, we can identify regions in which almost all carbon atoms become $s p^{3}$ bonded. Additionally, we observe a counteracting tendency for the carbon atoms to form shell-like substructures. To shift the balance between these two processes towards diamond nucleation strongly nonequilibrium conditions are required.
\end{abstract}

DOI: 10.1103/PhysRevB.63.081402

PACS number(s): 81.05.Tp, 81.10.Aj, 71.15.Ap

It has been discovered that fullerenes can form multilayered onion structures, when carbon soot is irradiated by electrons. ${ }^{1}$ Further electron or heavy ion irradiation causes the fullerenes to transform into diamond near their centers. ${ }^{2,3}$ The irradiation process is viewed to induce knockon displacements, collision cascades, and flux of $\mathrm{C}$ atoms towards the core, resulting in increase in the particle density, local pressure, and temperature. Finally, the conditions become preferable for the nucleation of diamond. It is necessary for the nucleation that the onion shells possess enough rigidity to withstand the increase in pressure and temperature in order to prevent a relaxation towards a graphitic core. Zaiser and Banhart ${ }^{4}$ have presented a thermodynamical quasiequilibrium theory to explain this irradiation-induced transformation to diamond. In this paper, we report results of atomic simulations of the collision-induced phenomena inside carbon fullerenes. These show the nucleation and growth of diamondlike structures.

The simulations are performed using the densityfunctional-based tight-binding method (DF-TB). ${ }^{5,6}$ The method has been successfully applied for studies of various systems in particular for carbon clusters such as small molecules, hydrocarbons, fullerenes, ${ }^{5}$ and extended allotropes such as amorphous and crystalline structures. ${ }^{7}$ Here we study the diamond nucleation process inside the smallest ideal carbon onion which consists of 300 atoms. This onion consists of two concentric fullerene shells, a $\mathrm{C}_{60}$ ball and a surrounding icosahedral $\mathrm{C}_{240}$ shell with average radii of 3.6 and $7.1 \AA$, respectively. We consider this low-energy allotrope as a likely core of real giant fullerenes and simulate the release of atoms due to knockon displacements in outer shells and their transport to this core by a sequence of random atom additions with zero initial velocities. The structural evolution is examined using a combination of molecular dynamics (MD) and a conjugate-gradient (CG) optimization technique and in dependence upon the number of (interstitial) atoms during an ad hoc injection.

To speed up the very demanding calculations, the fullerene core has originally been seeded with a ten-atomic cluster consisting of two interconnected five-membered rings. We find this atomic arrangement to be remarkably stable during an extensive phase-space search for likely atomic inclusions inside icosahedral $\mathrm{C}_{60} \cdot{ }^{8}$ We model the irradiation by randomly adding further interstitial carbon atoms to the fullerene. The number of atoms added are varied in order to minimize the influence of simulation details and initial settings upon the final result. We relax the growing structure between the additions to reflect that the relaxation rate is much higher than the damage creation rate. Finally, we refine our study by simulating the interstitial injection with an unseeded core. Note that the atom numbers given below always reflect the number of interstitial atoms added to either of the starting structures (i.e., the $\mathrm{C}_{10}$ seeded or the empty fullerene).

During simulation series A, 50 carbon atoms were initially placed $a d$ hoc into random locations between the $\mathrm{C}_{240}$ and seeded $\mathrm{C}_{60}$ shells. The relaxation was at first carried out by microcanonical MD for 240 fs with vanishing initial velocities. After equilibration the system reached a temperature of about $2100 \mathrm{~K}$ due to the atomic interactions. The system was then brought into contact with a heat bath and linearly cooled down to $1300 \mathrm{~K}$ with a rate of $0.69 \times 10^{15} \mathrm{~K} / \mathrm{s}$, using a simple velocity-scaling technique. Ten more atoms were added to random positions inside the fullerene. The addition was completed by microcanonical MD equilibration and thermalization at $2000 \mathrm{~K}$ for another $240 \mathrm{fs}$ and an annealing towards $1300 \mathrm{~K}$ using the same cooling rate as above. After another such injection cycle the total number of interstitials amounted to 70 . To simulate more intense irradiation and very rapid thermal dissipation to the surroundings, we continued the filling by adding sets of $20 \mathrm{C}$ atoms at random positions inside the structure. Again, we initially equilibrated the structure and thermalized it at $2000 \mathrm{~K}$ for $240 \mathrm{fs}$. Then, we CG relaxed the final snapshot structure of the hightemperature phase which yields a low-energy metastable configuration in phase space at $0 \mathrm{~K}$. The convergence criterion for the $\mathrm{CG}$ relaxations was a maximum rms force of 0.001 atomic units. We repeated such filling cycles until 150 extra atoms were placed into the fullerene. 
TABLE I. Hybridization and average coordination numbers vs number of incoming particles for irradiation molecular-dynamics simulation series A. The initial structure with 70 atoms added is formed by MD annealing as discussed in the text. Center refers to a core region with $8 \AA$ diameter.

\begin{tabular}{lcccc}
\hline \hline & $\begin{array}{c}s p^{2} \\
(\%)\end{array}$ & $\begin{array}{c}\text { Threefold nonplanar } \\
(\%)\end{array}$ & $\begin{array}{c}s p^{3} \\
(\%)\end{array}$ & $\begin{array}{c}\text { Average } \\
\text { coord. }\end{array}$ \\
\hline 70 added atoms & & & & \\
Whole structure & 63.8 & 20.4 & 11.1 & 3.07 \\
Center (28 atoms) & 50.0 & 17.9 & 28.6 & 3.29 \\
90 added atoms & & & & \\
Whole structure & 68.3 & 11.4 & 16.0 & 3.13 \\
Center (30 atoms) & 40.0 & 20.0 & 36.7 & 3.33 \\
110 added atoms & & & & \\
Whole structure & 55.8 & 15.5 & 25.8 & 3.23 \\
Center (34 atoms) & 11.8 & 20.6 & 67.6 & 3.68 \\
130 added atoms & & & & \\
Whole structure & 46.5 & 14.5 & 33.6 & 3.29 \\
Center (39 atoms) & 12.8 & 10.3 & 71.8 & 3.67 \\
\hline \hline
\end{tabular}

In the beginning of the simulation series $\mathbf{A}$ the $\mathrm{C}_{60}$ shell is destroyed and a larger shell-like structure consisting of $\sim 100$ atoms forms. This shell has a radius of about $4-5 \AA$ and initially leaves the outer $\mathrm{C}_{240}$ shell intact. This special atomic arrangement in the center appears to be quite stable under further injections. Clearly, a few atoms added avoid a location near the outermost $\mathrm{C}_{240}$ shell. The intershell distance between $\mathrm{C}_{240}$ and the emerging 100-atom shell is $\sim 2.5 \AA$, which could explain the experimentally observed intershell distance of $2.2 \AA$ in fullerene cores during early stages of irradiation. $^{2}$ After adding 70 atoms, the total fraction of fourfold-coordinated atoms is about $11 \%$. Upon increase of the atom number condensation takes not only place in the shell but also in the very core of the structure. The $s p^{3}$-bonded atoms appear at this stage rarely correlated at various places in the carbon network.

Continuing the filling with sets of 20 atoms leads to a breakdown of the interior shell structure. Since atoms added during these cycles do not have enough time and space to relax into fullerene-type arrangements, the condensation of fourfold-coordinated atoms is triggered. Interestingly, we can now identify an $s p^{3}$-rich cluster which binds to the outermost $\mathrm{C}_{240}$ shell. However, by the same time most of the inner atoms avoid the region near the outer shell indicating its stability due to $\pi$ bonding. The evolution of the model and its core cluster in terms of the number of three- and fourfold-coordinated atoms is presented in Table I and in Fig. 1. For an inner region with $8 \AA$ diameter, the $s p^{3}$ fraction appears to increase sharply for 90-110 atoms added.

As mentioned above, the diamond nucleation starts near the $\mathrm{C}_{240}$ shell which takes up defects and/or becomes bonded to the atomic arrangements in the core. As more atoms are added, $s p^{3}$-rich domains emerge and grow towards the center. There they fuse and form a highly fourfold-coordinated condensate as is indicated in Table I by the large fraction of

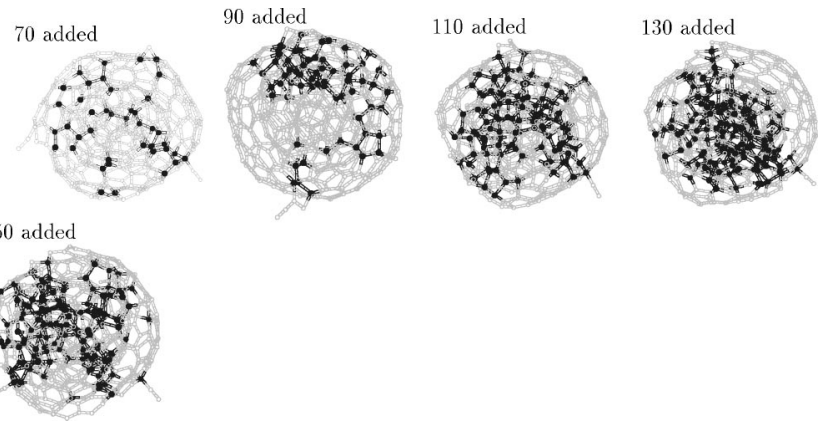

FIG. 1. The snapshots at the end of the the CG relaxation in simulation series $\mathbf{A}$. The $s p^{3}$ atoms are in dark color.

$s p^{3}$ atoms in the central areas of series $\mathbf{A}$ structures, see also Fig. 1. Figure 3 shows the total number of $s p^{3}$-hybridized atoms in the largest $s p^{3}$-bonded cluster during the addition. The neighboring atoms are considered to belong to a same cluster if they share a $s p^{3}$ bond.

While the total number of $s p^{3}$ bonded atoms declines slightly by about $5 \%$ when the filling is continued beyond 130 added atoms, the $s p^{3}$ concentration near the core region becomes saturated. This implies that the diamondlike structure, once nucleated, remains fairly stable under further injection of atoms even if the outer shell becomes disrupted.

The outermost shell of the fullerene develops a considerable number of defects during the irradiation simulation. These locally cause outward expansion of the shell, leading to pressure relief. Another mechanism to reduce strain in the core is emission of carbon dimers after their thermal activation. Both effects are contrary to diamond nucleation. However, in a real giant fullerene we expect that the manifold of fullerene layers supports the structural confinement of the core. Hence, the undesired stress relief effects become less important and the balance is shifted towards increase of the diamond yield.

The validity of this expectation was checked in a second simulation series $\mathbf{B}$, in which 70 and 110 carbon atoms, respectively, were introduced to random locations at once inside the seeded $\mathrm{C}_{300}$ onion structure. During this simulation

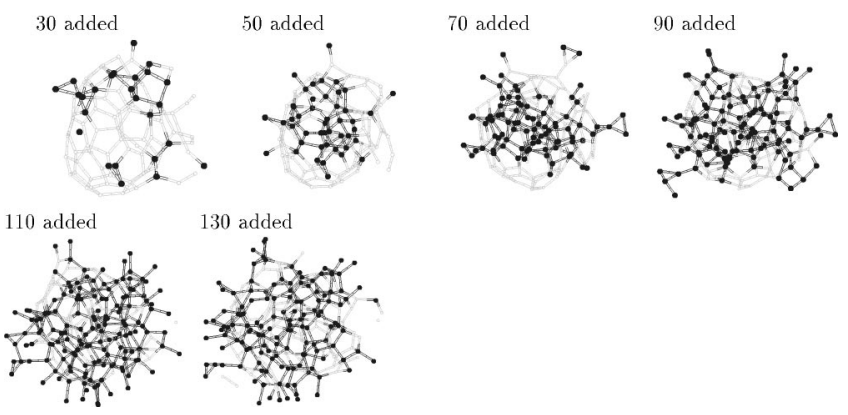

FIG. 2. The evolution of $s p^{3}$ atoms (dark color) during addition of carbon atoms in the CG-relaxation simulation series C. Shown are snapshots at the end of the CG relaxation. For clarity, the non$s p^{3}$ originally belonging to the outer $\mathrm{C}_{240}$ shell are left out from the picture. The topmost atom with 30 atoms added is on the surface of the $\mathrm{C}_{240}$ and from the 50 added atoms on the outermost atom locate on the surface of $\mathrm{C}_{240}$ shell. 
TABLE II. Hybridization and average coordination numbers vs number of incoming particles for irradiation simulation series $\mathbf{B}$ based on the two-step CG relaxation method. Center refers to a core region with $8 \AA$ diameter.

\begin{tabular}{lrccc}
\hline \hline & $\begin{array}{c}s p^{2} \\
(\%)\end{array}$ & $\begin{array}{c}\text { Threefold nonplanar } \\
(\%)\end{array}$ & $\begin{array}{c}s p^{3} \\
(\%)\end{array}$ & $\begin{array}{c}\text { Average } \\
\text { coord. }\end{array}$ \\
\hline 70 added atoms & & & & \\
Whole structure & 60.0 & 12.6 & 23.4 & 3.21 \\
Center (52 atoms) & 9.6 & 7.7 & 82.7 & 3.83 \\
& & & & \\
110 added atoms & & 17.9 & 41.0 & 3.40 \\
Whole structure & 37.6 & 3.1 & 93.8 & 3.94 \\
Center (64 atoms) & 3.1 & & & \\
\hline \hline
\end{tabular}

series, we CG relaxed the models in two steps. In the first step of the relaxation we fixed the positions of the outermost $\mathrm{C}_{240}$ shell. We then took the resulting structure as the starting point for a subsequent relaxation allowing all atoms to move freely. This simulation corresponds to an extreme nonequilibrium process with very rapid energy dissipation but puts additional constraints on the outer shell for stress relief. For the two-step relaxation with 70 added atoms the formation of diamondlike structures in the central region is about $83 \%$ and thus greater than the maximum value during simulation series A. Although the $s p^{3}$ content of the whole system is relatively small, the core part has many more $s p^{3}$-like atoms. For 110 atoms added the $s p^{3}$ fraction in the central area was the largest of all our models investigated. The percentage of $s p^{3}$-type atoms for the whole system and for a spherical central region are listed for this simulation series in Table II. In the model with 110 additional atoms we can identify small regions with radii of $3 \AA$ where the conversion to an amorphous diamond structure is almost completed. The radius of the outer $\mathrm{C}_{240}$ shell is increased by about $0.2 \AA$ for 70 and $0.4 \AA$ for 110 extra atoms added, respectively. Interestingly, both simulations of series $\mathbf{B}$ left the $\mathrm{C}_{240}$ shell almost undefected.

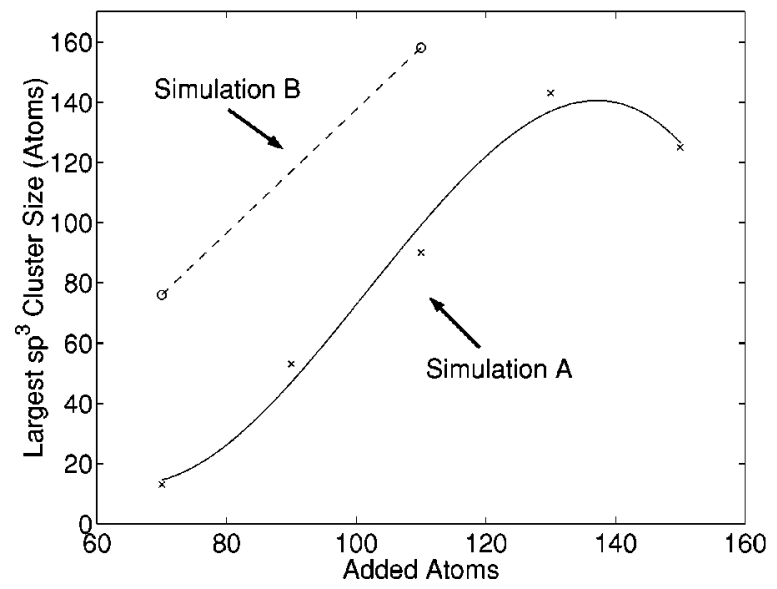

TABLE III. Hybridization and average coordination numbers vs number of incoming particles for irradiation simulation series $\mathbf{C}$ reflecting an atom-by-atom insertion. Center refers to a core region with $8 \AA$ diameter.

\begin{tabular}{lcccc}
\hline \hline & $\begin{array}{c}s p^{2} \\
(\%)\end{array}$ & $\begin{array}{c}\text { Threefold nonplanar } \\
(\%)\end{array}$ & $\begin{array}{c}s p^{3} \\
(\%)\end{array}$ & $\begin{array}{c}\text { Average } \\
\text { coord. }\end{array}$ \\
\hline 70 added atoms & & & & \\
Whole structure & 61.9 & 10.3 & 23.2 & 3.20 \\
Center (39 atoms) & 17.9 & 2.6 & 71.8 & 3.62 \\
& & & & \\
90 added atoms & & 13.8 & 31.0 & 3.30 \\
Whole structure & 53.1 & 5.0 & 72.5 & 3.73 \\
Center (40 atoms) & 22.5 & & & \\
& & & & \\
110 added atoms & & 15.9 & 38.0 & 3.36 \\
Whole structure & 43.2 & 9.8 & 68.3 & 3.66 \\
Center (41 atoms) & 19.5 & & & \\
& & & & \\
130 added atoms & & 7.3 & 68.3 & 3.66 \\
Whole structure & 46.0 & & & \\
Center (41 atoms) & 17.1 & & & \\
\hline \hline
\end{tabular}

In order to rule out a possible bias in the results due to the intial seeding or the setwise addition of atoms we, finally, performed another simulation series $\mathbf{C}$, in which the extra atoms were added one after another at random positions inside the unseeded $\mathrm{C}_{300}$. The whole $\mathrm{C}_{300+x}$ system was CG relaxed after each addition without any constraints. The number of fourfold-coordinated atoms saturates at about the same levels as for simulation series $\mathbf{A}(\sim 33 \%$ in total and $\sim 70 \%$ in the center). This indicates that the final results of the simulations remain nearly the same while using different simulation procedures (see also Table III).

However, at the early stages of the fullerene filling the structures differ in the simulations $\mathbf{A}, \mathbf{B}$, and $\mathbf{C}$. During the first steps of the simulation series $\mathbf{C}$ the the inner $\mathrm{C}_{60}$ shell becomes filled with chainlike structures rather than the more

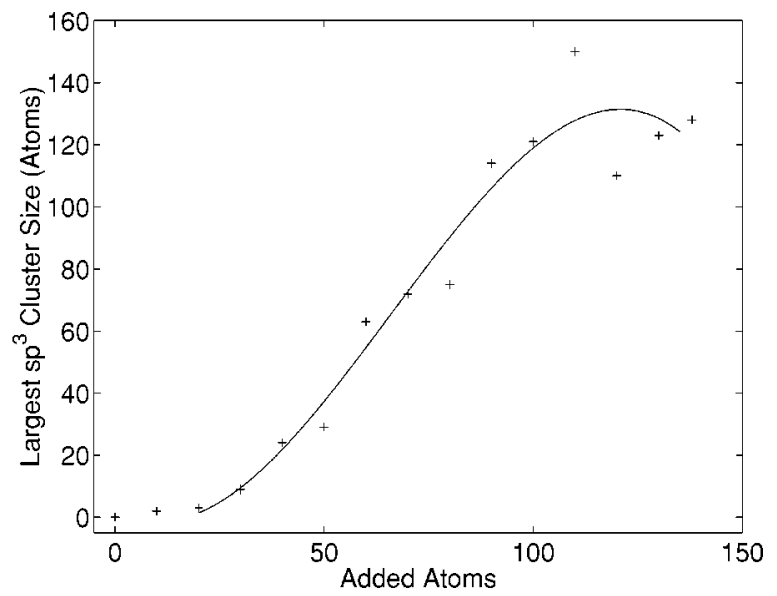

FIG. 3. Maximum size of clusters of interconnected atoms with $s p^{3}$ coordination in simulation series $\mathbf{A}, \mathbf{B}$ (left), and $\mathbf{C}$ (right). The number of randomly added atoms is on the $x$ axis. Crosses represent the evolution of the cluster size during series $\mathbf{A}$, circles correspond to series $\mathbf{B}$, and curves are only to guide the eye. 
stable cluster arrangement assumed in series A. Furthermore, the nucleation positions and growth directions of the $s p^{3}$ regions differ between simulation series $\mathbf{A}$ and $\mathbf{C}$. The evolution of the $s p^{3}$ region in simulation $\mathbf{C}$ is depicted in Fig. 2. The $s p^{3}$ atoms tend to aggregate as soon as they emerge. They start to form on the surface of the inner $\mathrm{C}_{60}$ shell by transformation of the $s p^{2}$-type atoms in the curved subsystem. Between 30 and 50 added atoms the $s p^{3}$ region has penetrated through the $\mathrm{C}_{60}$ fullerene. Between 50 and 70 added atoms the $s p^{3}$ cluster reaches to the surface of the $\mathrm{C}_{240}$ shell. After this more $s p^{3}$ atoms form in the middle of the cluster and between the shells and to some extent on surface of the $\mathrm{C}_{240}$. However, a major fraction of the $\mathrm{C}_{240}$ atoms remain $s p^{2}$ bonded. The evolution of the size of the largest $s p^{3}$ cluster is presented in Fig. 3. After the addition of 110 atoms the outermost $\mathrm{C}_{240}$ shell starts to break and can no longer maintain the pressure required for the diamond nucleation, resulting in decrease in the $s p^{3}$ contents.

Finally, we analyzed the bonding geometries of the central cores of the final snapshot $\mathrm{CG}$ relaxed system for the case of 110 atoms added from simulation series $\mathbf{A}$ and $\mathbf{C}$, and the CG relaxed systems with 70 and 110 atoms added from series $\mathbf{B}$. Bond angles and distances are characteristic of the diamond structure. For 70 added atoms in simulation series $\mathbf{B}$, the bond lengths are $1.48 \pm 0.05 \AA$. For 110 added atoms, the average bond lengths are $1.49 \pm 0.07 \AA, 1.52$ $\pm 0.07 \AA$, and $1.51 \pm 0.08$ for the simulation series $\mathbf{A}, \mathbf{B}$, and $\mathbf{C}$, respectively. The average angles are about $111 \pm 12$ degrees for the $\mathrm{CG}$ relaxed structures of series $\mathbf{A}$ and 112 \pm 13 degrees for series $\mathbf{C}$ but 109 \pm 12 degrees for the structures from series $\mathbf{B}$. However, the large deviations from the ideal diamond bonding indicate strong disorder which contradicts the experimental findings, where seemingly highly ordered diamond samples have been produced by the irradiation. ${ }^{3}$ We believe, however, that a transformation of the amorphous structure to a ordered crystal takes place on a longer timescale than can be studied with our present simulation techniques. The flux of incoming particles during the irradiation, which causes the temperature to be high in the core region of the fullerenes should cause the atoms to overcome the energy barriers associated with this transformation, while the confinement by the graphitic shells stabilizes the crystallite. We support the picture provided by Banhart and Ajayan, who propose an interstitial-vacancy self-annealing of the diamond nucleus and observe a very high stability of the emerging diamond crystallites. ${ }^{9}$

Ordered icosahedral molecules purely made of fourfoldcoordinated carbon have been discussed in the past as possible seeds for a diamond deposition, too. ${ }^{10}$ While free clusters of this type which include only 100 atoms can easily delaminate into concentric fullerenes, such structures appear to become stable against MD relaxation at $2700 \mathrm{~K}$ for atom numbers greater then $300 .{ }^{11}$ This together with our current results seems to indicate that there is a critical size for spherical atomic arrangements below which threefoldcoordinated atoms always dominate but above which diamondlike atoms can support themselves to bond into a completely fourfold-coordinated structure. The confinement by multilayer carbon fullerenes appears to lower this limit and to reduce the capabilities of $s p^{3}$-like atoms to relax back into a graphitic form. The nucleation in the carbon fullerene core involves a transition of quasi-two-dimensional curved shell structures into denser three-dimensionally packed diamondlike structures. Our simulation shows that this process requires rapid energy input by incoming particles along with ultrafast heat dissipation to the surroundings. We observe a transformation which is best described by a percolation of initially dispersed $s p^{3}$-bonded regions fusing after a certain threshold (the number of added atoms being between 50 and 100 atoms in our case) mostly as a one $s p^{3}$ cluster. Our simulation shows that atomic-scale details of the nucleation processes inside carbon fullerenes can be studied to a good extent by computational modeling using density-functionalbased interatomic forces.

This work has been supported by the Academy of Finland. We acknowledge the generous computer resources provided by Center for Scientific Computing (CSC) in Espoo, Finland.
*Present address: Department of Materials Science and Metallurgy, University of Cambridge, Pembroke Street, Cambridge CB2 3QZ, UK.

${ }^{1}$ D. Ugarte, Nature (London) 359, 707 (1992).

${ }^{2}$ F. Banhart and P.M. Ajayan, Nature (London) 382, 433 (1996).

${ }^{3}$ P. Wesolowski et al., Appl. Phys. Lett. 71, 1948 (1997).

${ }^{4}$ M. Zaiser and F. Banhart, Phys. Rev. Lett. 79, 3680 (1997).
${ }^{5}$ D. Porezag et al., Phys. Rev. B 51, 12947 (1995).

${ }^{6}$ C.M. Goringe et al., Rep. Prog. Phys. 60, 1447 (1997).

${ }^{7}$ Th. Frauenheim et al., J. Non-Cryst. Solids 182, 186 (1995).

${ }^{8}$ R. Astala et al. (unpublished).

${ }^{9}$ F. Banhart, J. Appl. Phys. 81, 3440 (1997).

${ }^{10}$ L. Zeger and T. Kaxiras, Phys. Rev. Lett. 70, 2920 (1993).

${ }^{11}$ G. Jungnickel et al., Phys. Status Solidi A 154, 109 (1996). 